\title{
Optimal Backlogging Over an Infinite Horizon Under Time Varying Convex Production and Inventory Costs
}

\author{
Archis Ghate • Robert L. Smith \\ Industrial Engineering, University of Washington, Box 352650, Seattle, Washington 98195, \\ $U S A$ \\ Industrial and Operations Engineering, University of Michigan, 1205 Beal Avenue, Ann \\ Arbor, Michigan 48109, USA \\ archis@u.washington.edu•rlsmith@umich.edu•
}

We consider an infinite horizon production planning problem with non-stationary, convex production and inventory costs. Backlogging is allowed unlike related previous work and inventory cost is interpreted as backlogging cost when inventory is negative. We create finite horizon truncations of the infinite horizon problem and employ classic results on convex production planning to derive a closed form formula for the minimum forecast horizon. We show that optimal production levels are monotonically increasing in the length of horizon leading to solution convergence and a rolling horizon procedure for delivering an infinite horizon optimal production plan. The minimum forecast horizon formula is employed to illustrate how cost parameters affect how far one must look into the future to make an infinite horizon optimal decision today.

\section{Introduction}

Production planning is a classic problem (Wagner and Whittin 1958, Denardo 1982, Federgruen and Tzur 1991) wherein one must decide how much of a product to produce in each period over a multi-period planning horizon so as to satisfy demand at minimum cost. An optimal production decision in the current period crucially depends on future demands and costs, which may vary with time due to technological change and other exogenous economic factors. As a result, in practice, a forecast of demands and costs has to be made and production be planned based on that forecast. Owing to considerable costs and difficulties involved in forecasting future data, a manufacturing firm prefers to use a short planning horizon. At the same time, the horizon must be long enough to mitigate the distortions introduced by 
end-of-study effects. The choice of planning horizon to employ is further complicated as the lifespan of the system under consideration is often indefinite and very long.

To circumvent these hurdles, production planning problems are often modeled over an infinite horizon. However, this presents a new difficulty especially when the data are nonstationary. Computing an infinite horizon optimal production decision in the first period itself may in general require infinite data rendering the task impossible. The question arises as to whether it is possible to somehow forecast a finite amount of data and compute a finite horizon optimal first period production level that remains infinite horizon optimal no matter what future demand and cost data are revealed to us. In that case, we could produce that optimal quantity in the first period and then roll forward to form a new infinite horizon problem with a new initial inventory on hand, repeating the procedure recursively ad infinitum to deliver an infinite horizon optimal production plan. This renders the infinite horizon problem solvable. Thus the key relevant issue is how much and which finite horizon data forecast is sufficient and necessary to compute an infinite horizon optimal first period production quantity, which relates to the notion of a minimum forecast horizon. This paper develops a closed form formula for the minimum forecast horizon for non-stationary production planning problems with convex production and inventory costs when backlogging is allowed.

Our approach is motivated by Smith and Zhang (1998) who provided closed-form upper bounds on minimum forecast horizons for similar problems without backlogging. This was achieved by establishing monotonicity of optimal first period decisions in horizon $N$. However, this type of monotonicity property fails to hold in the presence of backlogging, where the consideration of an additional period in the horizon can reveal an opportunity to produce in that period to satisfy an earlier demand leading to a decrease in the first period production. Interestingly, we are able to show that in the presence of certain natural assumptions, there exists a finite horizon version of the production planning problem in which monotonicity holds. We exploit monotonicity to obtain a closed-form formula for the minimum forecast horizon and employ it to perform numerical experiments to assess exactly how far into the future a production planner must look in order to make an infinite horizon optimal decision today.

The fundamental technical tool in our analysis is the work of Veinott (1964) who established that it is optimal to increase production in a single period in response to a unit increase in demand in a particular period. Smith and Zhang employed a special case of Veinott's 
result also presented in Denardo (1982) to prove existence and discovery of forecast horizons when backlogging is not allowed. Specifically, they embedded an $N$ horizon problem in an $N+1$ horizon problem by setting demand in the $N+1^{s t}$ period to 0 . Since it is optimal to end period $N$ with zero inventory in the $N$ horizon problem, the optimal production schedules for the $N$ horizon problem and the embedded $N+1$ horizon problem are the same. Then if we increase the demand in the $N+1^{s t}$ period to its actual value, one unit at a time, it is optimal to increase production levels unit by unit. This leads to the conclusion that increasing problem horizon does not decrease optimal production levels. Observe that the above statements fail if backlogging is allowed. This is because even though it may be optimal to end the $N$ horizon problem with zero inventory, it may no longer be optimal to end the $N^{\text {th }}$ period of the embedded problem with zero inventory. In particular, it may be optimal to carry negative inventory, i.e. to backorder in the $N^{t h}$ period of the embedded problem. Therefore, the simple device of embedding an $N$ horizon problem in an $N+1$ horizon problem fails when backlogging is allowed. Therefore, in this paper, we develop the new technique of embedding an $N$ horizon problem within a longer horizon problem, which enables us to exploit Veinott's general result to yield monotonicity even in the presence of backlogging. We first briefly discuss existing literature in this area.

The reader is referred to Chand et al. (2002) for an exhaustive survey of literature on forecast horizons. Early work in this area focussed on establishing existence of forecast horizons and techniques to discover them. Thompson and Sethi (1980) and Morton (1978a) assumed stationary linear costs, Kunreuther and Morton (1973) non-stationary linear costs whereas Kunreuther and Morton (1974), Morton (1978b), Modigliani and Hohn (1955), and Lee and Orr (1977) assumed stationary convex costs. Problems where production costs are fixed-plus-linear and inventory costs are linear have been studied extensively (Blackburn and Kunreuther 1974, Bean et al. 1987, Chand and Morton 1986, Chand et al. 1990, Chand et al. 1992, Eppen et al. 1969, Federgruen and Tzur 1994, Chen and Lee 1995, Zabel 1964, and other references in Chand et al. 2002). See Cheevaprawatdomrong and Smith (2004) for an extension of Smith and Zhang (1998) to the stochastic demand case with linear costs. There is also a body of literature that deals with computational procedures for solving finite horizon versions of the problem (Federgruen and Tzur 1991, Wagelmans et al. 1989). Researchers have investigated forecast horizon issues for doubly infinite convex programming problems in the past (Bean and Smith 1984, Bean and Smith 1993, Bes and Sethi 1988, and Schochetman and Smith 1989, Schochetman and Smith 1992). A common 
assumption in most of these works is that the infinite horizon optimal solution is unique, which is difficult to verify in practice and not always met. Crucially, we do not require this restrictive uniqueness assumption.

\section{A Class of Infinite Horizon Problems}

We will now define a class of production planning problems using the following generic notation: $D_{n}=$ the deterministic integer valued demand in period $\mathrm{n}, \alpha=$ discount factor for the time value of money $(0<\alpha<1), c_{n}(x)=$ the cost of producing $x$ units of product in period $\mathrm{n}, h_{n}(x)=$ the carrying and backlogging costs in period $n$ when its ending inventory level equals $x, P_{n}=$ the integer valued production level in period $\mathrm{n}$, and superscript * denotes optimality. Note that given a demand profile $D=\left(D_{1}, D_{2}, \ldots\right)$ and a production plan $P=\left(P_{1}, P_{2}, \ldots\right)$, the inventory profile $I=\left(I_{1}, I_{2}, \ldots\right)$ can be computed recursively as $I_{n}=I_{n-1}+P_{n}-D_{n}$, where the initial inventory $I_{0}$ is zero. Hence production quantities $P_{1}, P_{2}, \ldots$ are the only decision variables. An infinite horizon production planning problem is defined by an infinite sequence of data $\left\{\left(D_{n}, c_{n}(\cdot), h_{n}(\cdot)\right)\right\}_{n=1}^{\infty}$. We focus on data that satisfy the following assumptions.

Assumption 1. Production and inventory costs are convex, i.e., $c_{n}($.$) and h_{n}($.$) are convex$ functions with $c_{n}(0)=h_{n}(0)=0$ for all $n=1,2, \ldots$.

Assumption 2. The marginal backlogging costs are uniformly bounded from above and away from zero, i.e., $0<\pi \leq h_{n}\left(I_{n}-1\right)-h_{n}\left(I_{n}\right) \leq \beta<\infty$ for all non-positive integers $I_{n}$ and all $n=1,2, \ldots$

Assumption 3. There exists a finite cost production schedule.

Assumption 4. The marginal production costs are uniformly bounded from above and away from zero, i.e., $0<c_{n}\left(P_{n}\right)-c_{n}\left(P_{n}-1\right) \leq \gamma<\infty$ for all positive integers $P_{n}$ and all $n=1,2, \ldots$. The marginal cost of producing an additional unit in the first period is bounded from below by $\underline{c}_{1}>0$.

Assumption 5. The cheapest way to backlog one unit of demand forever is more expensive than the costliest way to produce that unit in the current period. That is,

$$
\gamma<\frac{\pi}{1-\alpha}
$$


Assumption 6. The marginal cost of carrying an additional unit of inventory is bounded from below by $\sigma$, i.e., by the convexity of $h_{n}(\cdot)$, we set $0 \leq \sigma \leq \inf _{n \geq 1} h_{n}(1)$.

The uniform upper bound $\gamma$ on the marginal cost of production in Assumption 4 is our most restrictive assumption. This uniform upper bound implies that the production cost functions 'track' a linear function and in particular restricts our results to uncapacitated problems. Our monotonicity and convergence results are likely to extend to the capacitated case following Veinott (1964), however, the derivation of closed form formulas for forecast horizons seems difficult if not impossible for capacitated problems, and specifically, for convex cost problems without this uniform bound. We use $\Phi$ to denote the class of time-varying data that satisfy the above assumptions, and the production planner is assumed to know parameters $\alpha, \beta, \pi, \gamma, \underline{c}_{1}, \sigma$ at the outset.

Given infinite horizon data $\left\{D_{n}, c_{n}(\cdot), h_{n}(\cdot)\right\}_{n=1}^{\infty} \equiv \phi \in \Phi$, the production planning problem is written as:

$$
\begin{gathered}
\left(Q^{\phi}\right) \min \sum_{n=1}^{\infty} \alpha^{n-1}\left[c_{n}\left(P_{n}\right)+h_{n}\left(I_{n}\right)\right] \\
\text { subject to } I_{n-1}+P_{n}-D_{n} \stackrel{=}{=} I_{n}, P_{n} \geq 0, P_{n}, I_{n} \text { integer, } n=1,2, \ldots
\end{gathered}
$$

Note any nonnegative integer production schedule is feasible to problem $\left(Q^{\phi}\right)$ and is denoted $P^{\phi}$ with $I^{\phi}$ the corresponding inventory schedule and $C^{\phi}$ the objective function value. However, there is a major hurdle: although any finite portion of data $\phi$ required to specify $\left(Q^{\phi}\right)$ can be forecast, it is impossible to know $\phi$ entirely. This obstacle can be overcome using the notion of rolling horizon solvability discussed in Section 1, which relies on the concept of forecast horizons discussed next.

\section{Finite Horizon Problems and Forecast Horizons}

In this section, we create a sequence of finite horizon truncations of the above infinite horizon problem. These truncations lead to a precise definition of forecast horizons for class $\Phi$. Properties of truncated problems are then exploited to calculate a simple formula for a forecast horizon.

\subsection{Finite Horizon Truncations}

Toward these goals, let $\infty>M=\min \left(\right.$ integer $\left.t \geq 0: \gamma<\frac{\pi\left(1-\alpha^{t+1}\right)}{1-\alpha}\right)$ where such an $M$ exists since $\frac{\pi\left(1-\alpha^{t+1}\right)}{1-\alpha}$ is monotonically increasing in $t$ with $\lim _{t \rightarrow \infty} \frac{\pi\left(1-\alpha^{t+1}\right)}{1-\alpha}=\pi /(1-\alpha)>\gamma$. 
It is thus strictly cheaper to produce a unit of demand than to backlog it for more than $M$ consecutive periods.

For some integer $\infty>N>0$, consider the following finite portion of some $\phi \in \Phi$ : demand $D_{n}$ in periods $n=1, \ldots, N$, production $\operatorname{costs} c_{n}(\cdot)$ in periods $n=1, \ldots, N+M$, and inventory costs $h_{n}(\cdot)$ in periods $n=1, \ldots, N+M$. Then we let $D(N)=(D_{1}, \ldots, D_{N}, \underbrace{0, \ldots, 0}_{M \text { times }})$ and use $\left(Q^{\phi}(N)\right)$ to denote the following problem:

$$
\begin{aligned}
\left(Q^{\phi}(N)\right) \min & \sum_{n=1}^{n=N+M} \\
I_{n-1}+P_{n}-D_{n}(N) & \alpha^{n-1}\left[c_{n}\left(P_{n}\right)+h_{n}\left(I_{n}\right)\right] \\
= & I_{n}, P_{n} \geq 0, P_{n}, I_{n} \text { integer, } n=1,2, \ldots, N+M .
\end{aligned}
$$

A production plan feasible for $\left(Q^{\phi}(N)\right)$ is denoted by $P^{\phi}(N)$ with $I^{\phi}(N)$ the corresponding inventory schedule and $C^{\phi}(\mathrm{N})$ the objective function value. Terminal inventory $I_{N+M}^{\phi}(N)$ is zero without loss of optimality. Observe that it is not optimal to have $I_{N+M}^{\phi}(N)>0$. Moreover, $I_{N+M}^{\phi}(N)<0 \Rightarrow I_{N}^{\phi}(N), \ldots, I_{N+M-1}^{\phi}(N)$ are also negative since $D_{N+1}(N), \ldots, D_{N+M}(N)$ are zero. But it is cheaper to produce in period $N$ rather than to backlog for $M+1$ consecutive periods.

For any horizon $N<\infty$ and $\phi \in \Phi$, we use $\phi(N)$ to denote the set of all infinite horizon data in $\Phi$ whose demands in the first $N$ periods and costs in the first $N+M$ periods match those in $\phi$.

Definition 7. A horizon $N_{f}<\infty$ is said to be a forecast horizon for class $\Phi$ if for every $\phi \in \Phi$, there exists a first period production optimal for $\left(Q^{\phi}\left(N_{f}\right)\right)$ that is also optimal for $\left(Q^{\theta}\right)$ for all $\theta \in \phi\left(N_{f}\right)$.

Definition 7 precisely delivers the key desirable property: if $N_{f}$ is known to be a forecast horizon at the outset, we can forecast demands for $N_{f}$ periods, costs for $N_{f}+M$ periods, compute an appropriate first period production level optimal for $\left(Q\left(N_{f}\right)\right)$, and rest assured that it is optimal for all possible infinite horizon continuations of demands beyond period $N_{f}$ and costs beyond period $N_{f}+M$, i.e., irrespective of the actual infinite horizon problem from $\Phi$ that we are confronting. More precisely, when $N_{f}$ is a forecast horizon according to Definition 7, we say that $N_{f}$ is the demand forecast horizon whereas $N_{f}+M$ is the cost forecast horizon. Importantly, such a forecast horizon, by definition, does not depend on a specific data stream from $\Phi$ but rather applies to any data stream from $\Phi$. We will derive a forecast horizon as a simple function of the parameters $\alpha, \beta, \pi, \gamma, \underline{c}_{1}, \sigma$, which can therefore be computed in advance of estimating any data. 


\subsection{Properties of Optimal Production Quantities}

Let $\left[P^{\phi}(1)\right]^{*}$ denote the lexicographically largest optimal production plan for $\left(Q^{\phi}(1)\right)$. Problem $\left(Q^{\phi}(N+1)\right)$ can be constructed from $\left(Q^{\phi}(N)\right)$ by (i) adding a terminal period $N+M+1$ with zero demand and then (ii) increasing demand in period $N+1$ from zero to $D_{N+1}$ one unit at a time. An optimal production plan for $\left(Q^{\phi}(N+1)\right)$ can hence be obtained from optimal production plan $\left[P^{\phi}(N)\right]^{*}$ for $\left(Q^{\phi}(N)\right)$ as follows. Production plan $\left(\left[P^{\phi}(N)\right]^{*}, 0\right)$ is optimal for the problem obtained after step (i). To see this, note by the argument in Section 3.1 that the ending inventory of period $N+M$ is zero, so that the ending inventory of period $N+M$ is non-positive. However, again by the same argument, it is not optimal for this ending inventory to be negative, implying that the production quantity in period $N+M+1$ equals zero. Finally if a strictly better plan than $\left[P^{\phi}(N)\right]^{*}$ exists for the first $N+M$ periods, this would contradict the optimality of $\left[P^{\phi}(N)\right]^{*}$ in $\left(Q^{\phi}(N)\right)$. Veinott $(1964)$ showed that given an optimal production plan for a demand profile in a finite horizon convex production planning problem with zero terminal inventory, it is optimal to respond to a unit increase in demand in any period by increasing production in some period by a unit. This result can be applied $D_{N+1}$ times to obtain the optimal production plan $\left[P^{\phi}(N+1)\right]^{*}$ from $\left(\left[P^{\phi}(N)\right]^{*}, 0\right)$ by unit increments of specific production volumes breaking ties by choosing the lexicographically largest plans. We conclude :

Proposition 8. The optimal production volumes $\left[P_{n}^{\phi}(N)\right]^{*}$ are monotonically increasing in $N$ for fixed $n, 1 \leq n \leq N+M$.

We investigate whether the first period production quantity $\left[P_{1}^{\phi}(N)\right]^{*}$ in problem $\left(Q^{\phi}(N)\right)$ (optimally) increases or remains the same as demand in period $N+1$ is increased one unit at a time starting at zero while constructing problem $\left(Q^{\phi}(N+1)\right)$. The next two propositions (proofs in the online companion) consider all alternatives to producing a unit of demand in period one and carrying inventory forward to period $N+1$ to resolve this issue. Specifically, for any non-negative real number $x$, letting $\lceil x\rceil$ denote the smallest integer strictly greater than $x$, we define for any integer $0 \leq R \leq M$,

$$
N(R) \equiv\left\lceil\log _{\alpha}\left(\frac{(1-\alpha) \underline{\mathrm{c}}_{1}+\sigma}{\sigma+\beta\left(1-\alpha^{R}\right)+(1-\alpha) \alpha^{R} \gamma}\right)\right\rceil .
$$

Proposition 9. When $N \geq N(R)$, it is strictly cheaper to produce in period $N+R+1$ to satisfy any unit of demand in period $N+1$ than producing in period 1 and carrying inventory forward to period $N+1$ in problem $\left(Q^{\phi}(N+1)\right)$ (proof in the online companion). 
Proposition 9 implies that $\left[P_{1}^{\phi}(N)\right]^{*}=\left[P_{1}^{\phi}(N+1)\right]^{*}$ for all $N \geq N(R)$.

Proposition 10. When $N \geq N(0)$, it is strictly cheaper to produce in period $N(0)+1$ to satisfy any unit of demand in period $N+1$ than producing in period 1 and carrying inventory forward to period $N+1$ in problem $\left(Q^{\phi}(N+1)\right)$ (proof in the online companion).

Proposition 10 implies that $\left[P_{1}^{\phi}(N)\right]^{*}=\left[P_{1}^{\phi}(N+1)\right]^{*}$ for all $N \geq N(0)$. In particular, these two propositions show that optimal production $\left[P_{1}^{\phi}(N)\right]^{*}$ remains locked at a fixed value for all sufficiently long finite horizon problems. The next question is which of the horizons in Equation (2) is the smallest. Simple algebra shows that if $\frac{\beta}{(1-\alpha)}>\gamma, N(R)$ is an increasing function of $R$. Since data for class $\Phi$ satisfy inequality $(1), \frac{\beta}{(1-\alpha)}>\gamma$ because $\beta$ is at least $\pi$. Hence $N(0)<N(R)$ for $1 \leq R \leq M$ and we have

$$
\left[P_{1}^{\phi}(N)\right]^{*}=\left[P_{1}^{\phi}(N(0))\right]^{*} \text { for all } N \geq N(0) .
$$

That is, for all finite horizon problems $Q^{\phi}(N)$ with $N \geq N(0)$, optimal production quantity $\left[P_{1}^{\phi}(N)\right]^{*}$ remains fixed at the value $\left[P_{1}^{\phi}(N(0))\right]^{*}$. We now show that $\left[P_{1}^{\phi}(N(0))\right]^{*}$ is also optimal for the infinite horizon problem $\phi$.

Assumptions 2, 3 and 4 can be used to show that the production volumes $\left[P_{n}^{\phi}(N)\right]^{*}$ and the corresponding inventory levels remain bounded (Lemma 17 in the online companion) and hence Proposition 8 implies that they converge monotonically upward to some $\left[\hat{P}_{n}^{\phi}\right]$ as $N \rightarrow \infty$. This in turn leads to value and then solution convergence as in Propositions 11 and 12 (proofs in the online companion).

Proposition 11. The optimal values $\left[C^{\phi}(N)\right]^{*}$ of problems $\left(Q^{\phi}(N)\right)$ converge to the optimal value $\left[C^{\phi}\right]^{*}$ of problem $\left(Q^{\phi}\right)$ as $N \rightarrow \infty$.

Proposition 12. The optimal production values $\left[P_{n}^{\phi}(N)\right]^{*}$ in problems $\left(Q^{\phi}(N)\right)$ converge monotonically upward to infinite horizon optimal production volumes $\left[P_{n}^{\phi}\right]^{*}$ in problem $\left(Q^{\phi}\right)$ as $N \rightarrow \infty$.

Since the optimal production volumes are integers, convergence implies that these volumes for sufficiently large horizons are infinite horizon optimal. Equation (3) then implies that $\left[P_{1}^{\phi}(N(0))\right]^{*}=\left[P_{1}^{\phi}\right]^{*}$ and $N(0)$ is a forecast horizon for class $\Phi$ by Definition 7 . 


\section{Minimum Forecast Horizons}

Owing to difficulties associated with forecasting future data, one is typically interested in the shortest possible forecast horizon for class $\Phi$. Clearly, the essential property of the minimum forecast horizon is that no shorter horizon is a forecast horizon in the sense of Definition 7 and is precisely captured as follows.

Definition 13. A forecast horizon $N_{f}^{*}$ for class $\Phi$ is called the minimum forecast horizon for $\Phi$ if for every horizon $H<N_{f}^{*}$, there exist $\phi \in \Phi$ and $\theta \in \phi(H)$ such that no first period production quantity optimal for $\left(Q^{\phi}(H)\right)$ is optimal for $\left(Q^{\theta}\right)$.

Thus, a minimum forecast horizon is the smallest forecast horizon that applies to any data stream.

Proposition 14. $N(0)$ given by Equation (2) is the minimum forecast horizon for class $\Phi$ (proof in the online companion).

We remark that the proof of this Proposition is constructive in that for any shorter horizon $H$, it demonstrates existence of data $\phi$ and $\theta \in \phi(H)$ such that no first period production level optimal for $Q^{\phi}(H)$ is optimal for $Q^{\theta}$.

We now illustrate how problem parameters impact the minimum forecast horizon. First notice that an annual interest rate of $r \%$ is related to a daily discount factor $\alpha$ through $\alpha=1 /\left(1+\frac{(r / 100)}{365}\right)$. We fix $r$ at $10 \%$ yielding $\alpha=0.999726102$. Suppose the costs are piecewise linear, convex and let $\gamma=u \underline{\mathrm{c}}_{1}$ for $u>1$, and $\sigma=v \underline{\mathrm{c}}_{1}$, and $\pi=x \underline{\mathrm{c}}_{1}$. Condition (1) is satisfied if $u<\left(1+\frac{365}{r}\right) x$, which reduces to $u<3651 x$ for $r=10 \%$. Then we have

$N(0)=\left\lceil\log _{\alpha}\left(\frac{(1-\alpha)+v}{v+(1-\alpha) u}\right)\right\rceil$. Sensitivity of demand forecast horizon $N(0)$ to $u$ and $v$, and of $M$ to $u$ and $x$ is illustrated in Figure 1. For any combination of $u, v, x, N(0)+M$ yields the cost forecast horizon.

\section{Acknowledgments}

This research was partially funded by the National Science Foundation under grant DMI0322114. The first author appreciates financial support from the University of Washington, Seattle during Summer 2007. We would like to thank the review team for their help in improving our manuscript. 

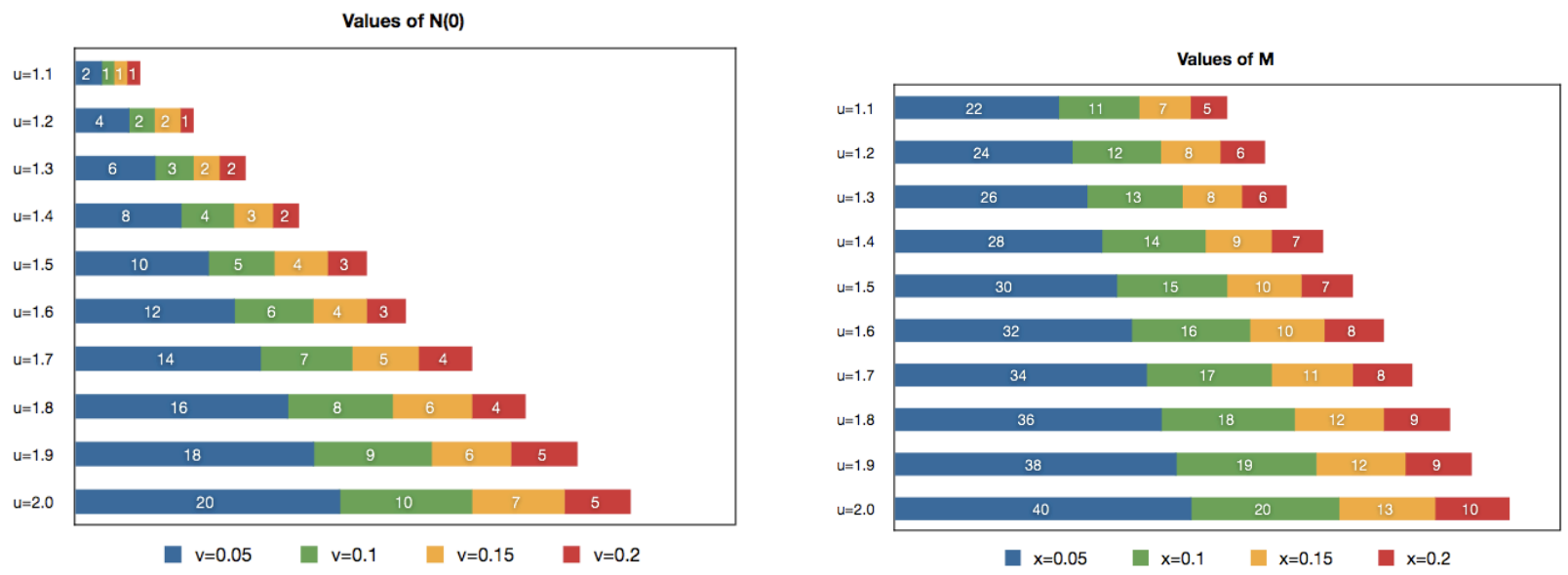

Figure 1: Sensitivity of $N(0)$ to $u$ and $v$ and of $M$ to $u$ and $x$ for $\alpha=0.999726102$.

\section{References}

Bean, J., Smith, R. L. 1984. Conditions for the Existence of Planning Horizons, Mathematics of Operations Research, 9, 391-401.

Bean, J., Smith, R. L. 1993. Conditions for the Discovery of Planning Horizons, Mathematical Programming, 59, 215 - 229.

Bean, J., Smith, R., C. Yano. 1987. Forecast Horizons for the Discounted Dynamic Lot Size Model Allowing Speculative Motive, Naval Research Logistics Quarterly, 34, 761-774.

Bes, C., Sethi, S. 1988. Concepts of forecast and decision horizons: Applications to Dynamic Stochastic Optimization Problems, Mathematics of Operations Research, 13, 295 - 310.

Blackburn, J., H. Kunreuther. 1974. Planning Horizons for the Dynamic Lot Size Model with Backlogging, Management Science, 21, 251-255.

Chand, S., Hsu, V. N., Sethi, S. 2002. Forecast, Solution, and Rolling Horizons in Operations Management Problems: A Classified Bibliography, Manufacturing \& Service Operations Management, 4 (1), $25-43$.

Chand, S., Morton, T. 1986. Minimal Forecast Horizon Procedures for Dynamic Lot Size Models, Naval Research Logistics Quarterly, 33, 111-122.

Chand, S., Sethi, S., Proth, J. M. 1990. Existence of Forecast Horizons in Undiscounted Discrete-Time Lot Size Models, Operations Research, 38(5), 884-892.

Chand, S., Sethi, S., Sorger, G. 1992. Forecast Horizons in the Discounted Dynamic Lot 
Size Model, Management Science, 38(7), 1034-1048.

Cheevaprawatdomrong, T., Smith, R. L. 2004. Infinite Horizon Production Scheduling in Time-varying Systems under Stochastic Demand, Operations Research, 52(1).

Chen, H. Lee., C. 1995. Error Bound for the Dynamic Lot Size Model Allowing Speculative Motive, IIE Transactions, 27, 683-688.

Denardo, E. 1982. Dynamic Programming : Models and Applications., Prentice Hall, Englewood Cliffs, New Jersey.

Eppen, G. D., Gould, F. J., Pashigian, B. P. 1969. Extensions of the Planning Horizon Theorem in the Dynamic Lot Size Model, Management Science, 15(5), 268-277.

Federgruen, A., Tzur, M. 1991. A Simple Forward Algorithm to Solve General Dynamic Lot Sizing Models with n Periods, Management Science, 15, 268 - 277.

Federgruen, A., Tzur, M. 1994. Minimal Forecast Horizons and a New Planning Procedure for the General Dynamic Lot Sizing Model: Nervousness Revisited, Operations Research, $42(3), 456-468$.

Kunreuther, H. C., Morton, T. E. 1973. Planning Horizons for Production Smoothing with Deterministic Demands, Management Science, 20, 110 - 125.

Kunreuther, H. C., Morton, T. E. 1974. General Planning Horizons for Production Smoothing with Deterministic Demands, Management Science, 20, 1037 - 1046.

Lee, D. R., Orr, D., 1977. Further Results on Planning Horizons in the Production Smoothing Problem, Management Science, 23, 490 - 498.

Modigliani, F., Hohn, F. E. 1955. Production Planning Over Time and the Nature of the Expectation and Planning Horizon, Econometrica, 23, 46 - 66.

Morton, T. 1978a. The Non-stationary Infinite Horizon Inventory Problem, Management Science, 24, 1474 - 1482.

Morton, T. 1978b. Universal Planning Horizons for Generalized Convex Production Scheduling, Operations Research, 26, 1046 - 1058.

Schochetman, I. E., Smith, R. L. 1989. Infinite Horizon Optimization, Mathematics of Operations Research, 14, 559 -574.

Schochetman, I. E., Smith, R. L. 1992. Finite Dimensional Approximation in Infinite Dimensional Mathematical Programming, Mathematical Programming, 54, 307 - 333. 
Smith, R. L., Zhang, R. 1998. Infinite Horizon Production Planning in Time-varying Systems with Convex Production and Inventory Costs, Management Science, 44 (9), 1313 - 1320.

Thompson, L. J., Sethi, S. P. 1980. Turnpike Horizons for Production Planning, Management Science, 26, 229 - 241.

Veinott, A. F., Jr. 1964. Production Planning with Convex Costs : A Parametric Study, Management Science, 10, 441 - 460.

Wagelmans, A., Van Hoesel, S., Kolen, A. 1989. Economic Lot Sizing : An $O(n \log n)$ Algorithm that Runs in Linear Time in the Wagner-Whitin case, CORE discussion paper no. 8922, Universite Catholique de Louvain, Louvain-la-Neuve, Belgium.

Wagner, H.M., Whitin, T. M. 1958. Dynamic Version of the Economic Lot Size Model, Management Science, 5, 89-96.

Zabel, E. 1964. Some Generalizations of an Inventory Planning Horizon Theorem, Management Science, 10(3), 465-471. 


\section{Online Companion}

Statements and proofs of technical results are provided in this online companion.

\section{Proof of Proposition 9}

Proof. Let $N \geq N(R)$. Since $0<\alpha<1$, we have

$$
\begin{gathered}
\alpha^{N}\left((1-\alpha) \alpha^{R} \gamma+\sigma+\beta\left(1-\alpha^{R}\right)\right)<\left((1-\alpha) \underline{\mathrm{c}}_{1}+\sigma\right) \\
(1-\alpha) \gamma \alpha^{N+R}+\alpha^{N} \beta\left(1-\alpha^{R}\right)<\left((1-\alpha) \underline{\mathrm{c}}_{1}+\sigma\left(1-\alpha^{N}\right)\right) \\
\gamma \alpha^{N+R}+\beta \frac{\alpha^{N}\left(1-\alpha^{R}\right)}{(1-\alpha)}<\underline{\mathrm{c}}_{1}+\frac{\sigma\left(1-\alpha^{N}\right)}{(1-\alpha)}=\underline{\mathrm{c}}_{1}+\sum_{n=1}^{N} \alpha^{n-1} \sigma \\
\gamma \alpha^{N+R}+\sum_{n=N+1}^{N+R} \alpha^{n-1} \beta<\underline{\mathrm{c}}_{1}+\sum_{n=1}^{N} \alpha^{n-1} \sigma
\end{gathered}
$$

Thus, the most expensive way of producing one unit in period $N+R+1$ and backlogging at the end of periods $N+1, N+2, \ldots, N+R$ is strictly cheaper than the least expensive way of producing that unit in the first period and carrying it forward in the inventory. Then, any way of producing one unit in period $N+R+1$ and backlogging at the end of periods $N+1, N+2, \ldots, N+R$ must be strictly cheaper than any way of producing that unit in the first period and carrying it forward in the inventory. This concludes the proof.

\section{Proof of Proposition 10}

Proof. Notice that the case $N=N(0)$ is already considered in Proposition 9. Therefore, we only consider $N>N(0)$. No matter whether we produce in period 1 or period $N(0)+1$, we have to carry inventory forward from period $N(0)+1$ to period $N+1$ (if we produce in period 1, we also have to carry inventory forward from period 1 to period $N(0)+1)$. We will show that the cost of carrying any unit of inventory from period one to period $N(0)+1$ is strictly more than the cost of producing that unit in period $N(0)+1$. Note that since $0<\alpha<1$,

$$
\begin{aligned}
\frac{(1-\alpha) \underline{\mathrm{c}}_{1}+\sigma}{\gamma(1-\alpha)+\sigma}>\alpha^{N(0)}, \text { i.e., }(1-\alpha) \underline{\mathrm{c}}_{1}+\left(1-\alpha^{N(0)}\right) \sigma>\gamma \alpha^{N(0)}(1-\alpha), \text { i.e., } \\
\underline{\mathrm{c}}_{1}+\frac{\left(1-\alpha^{N(0)}\right) \sigma}{(1-\alpha)}>\gamma \alpha^{N(0)}, \text { i.e., } \underline{\mathrm{c}}_{1}+\sum_{n=1}^{N(0)} \alpha^{n-1} \sigma>\gamma \alpha^{N(0)} .
\end{aligned}
$$


Thus, the cheapest way of producing one unit in period 1 and carrying inventory forward to period $N(0)+1$ is more expensive than the most expensive way of producing that unit in period $N(0)+1$. Therefore, any way of producing one unit in period $N(0)+1$ must be cheaper than any way of producing that unit in period 1 and carrying inventory forward to period $N(0)+1$.

Proof of Proposition 11 requires Lemmas 15, 16, 17.

Lemma 15. $\left[C^{\phi}(N)\right]^{*} \leq\left(C^{\phi}\right)^{*}$, i.e., the optimal cost of problem $\left(Q^{\phi}(N)\right)$ is less than or equal to the optimal cost of the infinite horizon problem $\left(Q^{\phi}\right)$. Similarly, $\left[C^{\phi}(N)\right]^{*}$ is monotonically increasing in $N$.

Proof. Assume on the contrary that $\left(C^{\phi}\right)^{*}<\left[C^{\phi}(N)\right]^{*}$. Let $\left(P^{\phi}\right)^{*}$ be an infinite horizon production schedule optimal for $\left(Q^{\phi}\right)$ with $\left(I^{\phi}\right)^{*}$ the corresponding inventory schedule. We modify $\left(P^{\phi}\right)^{*}$ to construct a production schedule (say) $P^{\phi}(N)$ feasible to problem $Q^{\phi}(N)$ with corresponding inventory schedule $I^{\phi}(N)$ with total production and inventory $\operatorname{cost} C^{\phi}(N) \leq$ $\left(C^{\phi}\right)^{*}$. We consider three cases:

1. Case 1: $\left(I_{N}^{\phi}\right)^{*}=0$. Then set $P_{n}^{\phi}(N)=\left(P_{n}^{\phi}\right)^{*}$ for $n=1, \ldots, N$, and $P_{n}^{\phi}(N)=0$ for $N+1 \leq n \leq N+M$. Then the total production plus inventory cost of schedule $P^{\phi}(N)$ in the first $N$ periods of $Q^{\phi}(N)$ is equal to that of $\left(P^{\phi}\right)^{*}$ in $Q^{\phi}$. On the other hand, the total cost of $P^{\phi}(N)$ in periods after $N$ in $Q^{\phi}(N)$ is zero, i.e., at most that of $\left(P^{\phi}\right)^{*}$ in $Q^{\phi}$. Specifically, $C^{\phi}(N) \leq\left(C^{\phi}\right)^{*}$.

2. Case 2: $\left(I_{N}^{\phi}\right)^{*}>0$. Let $T \leq N$ be the earliest period with the property that $\left(I_{n}^{\phi}\right)^{*}>0$ for all $n=T, \ldots, N$ (existence of such a period is guaranteed because $N$ has this property). Set $P_{n}^{\phi}(N)=\left(P_{n}^{\phi}\right)^{*}$ for $n=1, \ldots, T-1$, and $P_{n}^{\phi}(N)=0$ for $N+1 \leq n \leq$ $N+M$. However, for $n=T, \ldots, N$, set $P_{n}^{\phi}(N)$ by reducing production levels in (some of the) corresponding periods from schedule $\left(P^{\phi}\right)^{*}$ so that $I_{N}^{\phi}(N)=0$. Then the total production plus inventory cost of schedule $P^{\phi}(N)$ in the first $T-1$ periods of $Q^{\phi}(N)$ is equal to that of $\left(P^{\phi}\right)^{*}$ in $Q^{\phi}$. Moreover, the total production plus inventory cost of schedule $P^{\phi}(N)$ in periods $T$ through $N$ of $Q^{\phi}(N)$ is at most that of $\left(P^{\phi}\right)^{*}$ in $Q^{\phi}$. Finally, the total cost of schedule $P^{\phi}(N)$ in periods after $N$ in $Q^{\phi}(N)$ is zero, i.e., at most that of $\left(P^{\phi}\right)^{*}$ in $Q^{\phi}$. Thus, $C^{\phi}(N) \leq\left(C^{\phi}\right)^{*}$. 
3. Case 3: $\left(I_{N}^{\phi}\right)^{*}<0$. Let $T \geq N$ be the latest period with the property that $\left(I_{n}^{\phi}\right)^{*}<0$ for all $n=N, \ldots, T \leq N+M$ (existence of such a period is guaranteed because $N$ has this property). Set $P_{n}^{\phi}(N)=\left(P_{n}^{\phi}\right)^{*}$ for $n=1, \ldots, N$ and $P_{n}^{\phi}(N)=0$ for $n \geq T+2$. However, for $n=N+1, \ldots, T+1$, set $P_{n}^{\phi}(N)$ by reducing production levels in (some of the) corresponding periods from schedule $\left(P^{\phi}\right)^{*}$ so that $I_{T+1}^{\phi}(N)=0$. Then the total production plus inventory cost of schedule $P^{\phi}(N)$ in first $N$ periods of $Q^{\phi}(N)$ is equal to that of $\left(P^{\phi}\right)^{*}$ in $Q^{\phi}$. Moreover, the total production plus inventory cost of schedule $P^{\phi}(N)$ in periods $N+1$ through $T+1$ of $Q^{\phi}(N)$ is at most that of $\left(P^{\phi}\right)^{*}$ in $Q^{\phi}$. Finally, the total cost of schedule $P^{\phi}(N)$ in periods after $T+1$ in $Q^{\phi}(N)$ is zero, i.e., at most that of $\left(P^{\phi}\right)^{*}$ in $Q^{\phi}$. Thus, $C^{\phi}(N) \leq\left(C^{\phi}\right)^{*}$.

Thus, we have, $C^{\phi}(N) \leq\left(C^{\phi}\right)^{*}<\left[C^{\phi}(N)\right]^{*}$ contradicting optimality of cost $\left[C^{\phi}(N)\right]^{*}$ in $\left(Q^{\phi}(N)\right)$. This proves the first claim. The second claim can also be proved similarly.

Lemma 16. The optimal values of $\left(Q^{\phi}(N)\right)$ and $\left(Q^{\phi}\right)$ satisfy $\lim _{N \rightarrow \infty}\left[C^{\phi}(N)\right]^{*} \leq\left(C^{\phi}\right)^{*}$.

Proof. Recall that $\left[C^{\phi}(N)\right]^{*}$ is monotonically increasing in $N$ from Lemma 15. Further, $\left[C^{\phi}(N)\right]^{*} \leq\left(C^{\phi}\right)^{*}<\infty$ by Lemma 15 and Assumption 3. Therefore the limit on the left hand side is finite and satisfies the said inequality.

Lemma 17. There exist finite production bounds $\bar{P}_{n}^{\phi}$, so that $\left[P_{n}^{\phi}(N)\right]^{*} \leq \bar{P}_{n}^{\phi}<\infty$ for all $N$. Similarly for $\left|\left[I_{n}^{\phi}(N)\right]^{*}\right|$.

Proof. Suppose not. Then there exists some $n$ and subsequence $N_{k}^{n}, k=1,2 \ldots$ such that $\left[P_{n}^{\phi}\left(N_{k}^{n}\right)\right]^{*} \rightarrow \infty$ as $k \rightarrow \infty$. Hence, by Assumption $4, c_{n}\left(\left[P_{n}^{\phi}\left(N_{k}^{n}\right)\right]^{*}\right) \rightarrow \infty$ as $k \rightarrow \infty$. This implies that $\left[C^{\phi}\left(N_{k}^{n}\right)\right]^{*} \rightarrow \infty$ as $k \rightarrow \infty$. However, $\left[C^{\phi}\left(N_{k}^{n}\right)\right]^{*} \leq\left(C^{\phi}\right)^{*}<\infty$, where the first inequality follows from Lemma 15 and the second by Assumption 3. This leads to a contradiction. Bounds on the production levels also lead to bounds on inventory levels when they are non-negative. The conclusion that backlogged inventory also remains bounded can be reached by repeating the above argument with the help of Assumptions 2 and 3 .

\section{Proof of Proposition 11}

Proof. Since the production schedules $\left[P^{\phi}(N)\right]^{*}$ (and the inventory schedules $\left.\left[I^{\phi}(N)\right]^{*}\right)$ converge componentwise to some $\hat{P}^{\phi}$ (and $\hat{I}^{\phi}$ ), and the cost function is continuous, $\left[C^{\phi}(N)\right]^{*}$ converges to some real number $\hat{C}^{\phi} \equiv C\left(\hat{P}^{\phi}\right)$, the cost of production schedule $\hat{P}^{\phi}$. That is, 
$\lim _{N \rightarrow \infty}\left[C^{\phi}(N)\right]^{*}=\hat{C}^{\phi}$. In view of Lemma 16, $\hat{C}^{\phi} \leq\left(C^{\phi}\right)^{*}$. We are done if $\hat{C}^{\phi}=\left(C^{\phi}\right)^{*}$. Therefore, consider the case $\hat{C}^{\phi}<\left(C^{\phi}\right)^{*}$. Observe that the schedule $\hat{P}^{\phi}$ is infinite horizon feasible. Hence, $\hat{C}^{\phi}<\left(C^{\phi}\right)^{*}$ implies that the cost of a feasible production schedule is strictly less than the optimal cost, which is a contradiction. Therefore, $\hat{C}^{\phi}=\left(C^{\phi}\right)^{*}$.

\section{Proof of Proposition 12}

Proof. Consider any positive integer $R$.

$$
\begin{gathered}
\sum_{n=1}^{R} \alpha^{n-1}\left[c_{n}\left(\hat{P}_{n}^{\phi}\right)+h_{n}\left(\hat{I}_{n}^{\phi}\right)\right]=\lim _{N \rightarrow \infty} \sum_{n=1}^{R} \alpha^{n-1}\left[c_{n}\left(\left[P_{n}^{\phi}(N)\right]^{*}\right)+h_{n}\left(\left[I_{n}^{\phi}(N)\right]^{*}\right)\right] \\
\leq \lim _{N \rightarrow \infty} \sum_{n=1}^{N+M} \alpha^{n-1}\left[c_{n}\left(\left[P_{n}^{\phi}(N)\right]^{*}\right)+h_{n}\left(\left[I_{n}^{\phi}(N)\right]^{*}\right)\right] \\
=\lim _{N \rightarrow \infty}\left[C^{\phi}(N)\right]^{*} \leq\left(C^{\phi}\right)^{*}
\end{gathered}
$$

where the last inequality follows from Lemma 16. Now taking the limit as $R \rightarrow \infty$ on both sides

$$
C\left(\hat{P}^{\phi}\right) \equiv \lim _{R \rightarrow \infty} \sum_{n=1}^{R} \alpha^{n-1}\left[c_{n}\left(\hat{P}_{n}^{\phi}\right)+h_{n}\left(\hat{I}_{n}^{\phi}\right)\right] \leq\left(C^{\phi}\right)^{*} .
$$

Since $\hat{P}^{\phi}$ is feasible to $\left(Q^{\phi}\right)$, the above inequality implies that it is also optimal.

\section{Proof of Proposition 14}

Proof. Let $H<N(0)$ be any finite horizon. We construct forecast $\phi \in \Phi$ and $\theta \in \phi(H)$ such that no first period optimal decision to problem $\left(Q^{\phi}(H)\right)$ is optimal for $\left(Q^{\theta}\right)$. Let $\phi$ be such that (i) production cost in the first period is linear with cost/unit produced $=\underline{\mathrm{c}}_{1}$, (ii) production cost in all other periods is also linear with cost/unit produced $=\gamma$, (iii) inventory cost in all periods is linear with cost/unit inventory $=\sigma$, (iv) backlogging cost in all periods is linear with cost/unit backlogged $=\beta$, and $(\mathrm{v})$ the demand in every period is one unit. Clearly, $\phi \in \Phi$. Moreover, since $\beta+\alpha \gamma>\gamma$, it is strictly cheaper to produce in period $n$ than backlogging in period $n$ and producing in period $n+1$ for any $n>1$. In fact, it is strictly cheaper to produce in period $n$ than backlogging in periods $n, n+1, \ldots, n+r$ and producing in period $n+r+1$ for any $r \geq 0$ and any $n>1$. To see this, observe that the 
cost of the latter is

$$
\begin{aligned}
\alpha^{n-1}\left(\beta+\alpha \beta+\alpha^{2} \beta+\ldots+\alpha^{r} \beta+\alpha^{r+1} \gamma\right) & =\alpha^{n-1}\left(\beta+\alpha \beta+\alpha^{2} \beta+\ldots+\alpha^{r}(\beta+\alpha \gamma)\right) \\
>\alpha^{n-1}\left(\beta+\alpha \beta+\alpha^{2} \beta+\ldots+\alpha^{r} \gamma\right) & =\alpha^{n-1}\left(\beta+\alpha \beta+\alpha^{2} \beta+\ldots+\alpha^{r-1}(\beta+\alpha \gamma)\right) \\
\ldots \ldots & =\alpha^{n-1}(\beta+\alpha \gamma)>\alpha^{n-1} \gamma
\end{aligned}
$$

which is the cost of the former. Let $x^{*}$ be any first period production level optimal for $\left(Q^{\phi}(H)\right)$. Now consider a forecast $\theta \in \phi(H)$ where demand in the first $H+1$ periods is one and demand in all periods beyond $H+1$ is zero. The above discussion implies that it is strictly cheaper to produce in period $H+1$ than backlogging and producing in any period beyond $H+1$. In addition, $H<N(0)$ implies that $\underline{\mathrm{c}}_{1}+\sum_{n=1}^{N} \alpha^{n-1} \sigma<\alpha^{N} \gamma \forall N \leq H$. It is thus even cheaper to produce in period one and carry inventory forward to period $H+1$ than producing in period $H+1$. Hence, the (strictly) cheapest way to satisfy unit demand in period $H+1$ is to produce in period one and carry inventory forward. Thus, $H$ cannot be a forecast horizon implying that $N(0)$ is the minimum forecast horizon. 